\title{
Seven- to eight-year follow-up of the CoolCap trial of head cooling for neonatal encephalopathy
}

\author{
Ronnie Guillet' ${ }^{1}$ A. David Edwards², Marianne Thoresen ${ }^{3}$, Donna M. Ferriero ${ }^{4}$, Peter D. Gluckman ${ }^{5}$, Andrew Whitelaw ${ }^{6}$ \\ and Alistair Jan Gunn?; on behalf of the CoolCap Trial Group
}

INTRODUCTION: We sought to determine whether 18- to 22-mo neurodevelopmental outcomes predicted functional outcomes at 7-8 y for survivors of the CoolCap study of therapeutic hypothermia for neonates with hypoxic-ischemic encephalopathy.

RESULTS: WeeFIM ratings were completed at 7-8 y of age on 62 (32 cooled; 30 standard care) of 135 surviving children who had had neurodevelopmental assessment at 18 mo. There was 1 refusal, 58 lost to follow-up, and 14 children whose centers declined to participate. Disability status at 18 mo was strongly associated with WeeFIM ratings $(P<0.001)$; there was no significant effect of treatment $(P=0.83)$.

DISCUSSION: Functional outcome at 7-8 y of survivors of neonatal encephalopathy is associated with 18-mo neurodevelopmental assessment, supporting the long-term predictive value of a favorable outcome at 18 mo assessed by published trials of therapeutic hypothermia.

METHODS: All surviving children who participated in the CoolCap study and were assessed at 18 mo were eligible for reassessment using the WeeFIM instrument that qualitatively measures self-care, mobility, and cognitive function. Center investigators obtained consent from the families for a certified researcher to administer the WeeFIM instrument by phone.

$\mathbf{M}^{2}$ oderate to severe neonatal encephalopathy continues to be an important cause of acute neurologic injury at birth and subsequent long-term disability, affecting $\sim 2-3$ cases per 1,000 term live births in developed countries (1) and many more in less developed nations (2). There is now compelling evidence that induced mild to moderate cerebral hypothermia can reduce neurodevelopmental handicap and improve survival without disability at 18 mo (3). Both head and whole body cooling seem to provide similar levels of protection (3). For example, the CoolCap trial, a randomized, multicenter, controlled trial of head cooling combined with mild total body hypothermia for neonatal encephalopathy, suggested that hypothermia reduced disability in infants with less severe encephalopathy $(4,5)$. At present, we do not know whether the benefits of cooling are sustained or whether induced hypothermia reduces cognitive or milder motor disabilities later in childhood (6). Infants who had hypoxic-ischemic encephalopathy at birth may have no disability on examination at 18-24 mo of age but go on to develop more subtle cognitive and motor neurological disabilities at school age $(7,8)$.

Study of the infants entered into the CoolCap trial provides an opportunity to learn about the early childhood sequelae of hypoxic-ischemic encephalopathy and cerebral cooling. We sought to reassess these children at early school age (7-8 y) to determine whether assessment at 18 mo of age predicted functional improvement in later childhood.

\section{RESULTS \\ Study Population}

As shown in Figure 1, of 234 infants enrolled in the CoolCap study, 83 had died (38 cooled and 45 noncooled) by 7-8 y, and 16 children were not assessed at 18 mo (Figure 1). Of 135 surviving children who had had 18-mo neurodevelopmental assessment, 62 (46\%) were assessed at 7-8 y of age, 58 children were lost to follow-up, the centers declined to participate for 14 children, and 1 family refused.

The baseline characteristics and the 18- to 22-mo outcomes of the 62 tested children did not apparently differ from those of survivors who were not assessed at 7-8 y of age (Tables 1 and 2). There were no differences in baseline characteristics between the treatment groups for children assessed at 7-8 y of age (Table 3$)$, although there was a trend $(P=0.08)$ for more of the cooled subjects to have greater severity of clinically assessed encephalopathy at birth.

\section{WeeFIM Results}

Outcome at 18 mo was strongly associated with overall WeeFIM ratings at $7-8$ y of age $(P<0.001$, logistic regression; Figure 2; Table 4), with a rating of $115 \pm 19$ (mean \pm SD) in those with favorable 18-mo outcomes and $67 \pm 42$ in those with adverse outcomes. Adjustment for baseline variables and treatment group did not affect this association $(P<0.001)$,

\footnotetext{
'Department of Pediatrics, University of Rochester Medical Center, Rochester, New York; ${ }^{2}$ Centre for the Developing Brain, Imperial College, London, UK; ${ }^{3}$ St. Michael's Hospital, University of Bristol, Bristol, UK; ${ }^{4}$ Department of Neurology and Department of Pediatrics, University of California, San Francisco, San Francisco, California; ${ }^{5}$ The Liggins Institute, University of Auckland, Auckland, New Zealand; ${ }^{6}$ Southmead Hospital, University of Bristol, Bristol, UK; ${ }^{7}$ Department of Physiology and Department of Paediatrics, University of Auckland, Auckland, New Zealand. Correspondence: Ronnie Guillet

(ronnie_guillet@urmc.rochester.edu)
} 


\section{Articles | CoolCapstudy follow-up}

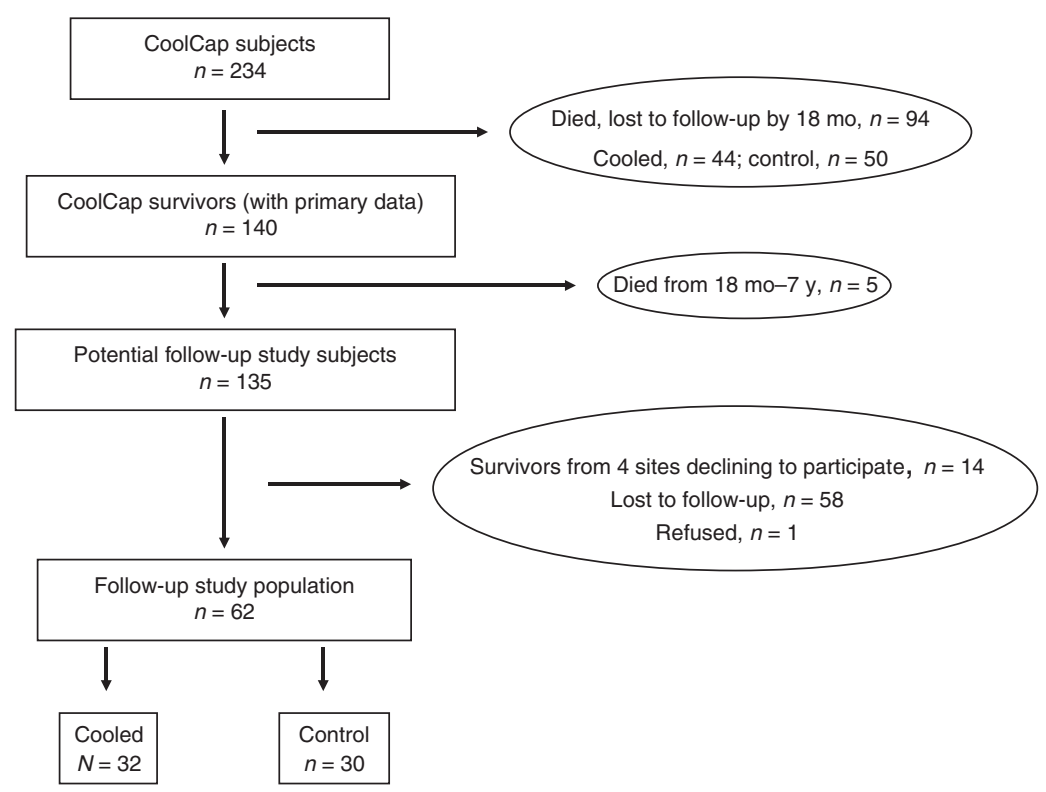

Figure 1. Study population: Children eligible for this study were survivors of the CoolCap study cared for originally in centers whose site investigator agreed to participate in the follow-up study.

Table 1. Demographic data for tested and untested survivors with 18-mo data

\begin{tabular}{|c|c|c|c|c|}
\hline & $\begin{array}{l}\text { Tested } \\
(N=62)\end{array}$ & $\begin{array}{l}\text { Not tested } \\
(N=73)\end{array}$ & $\begin{array}{l}\text { All survivors } \\
(N=135)\end{array}$ & $P$ value \\
\hline Birth weight (g) & $3,386 \pm 698$ & $3,416 \pm 711$ & $3,402 \pm 702$ & 0.81 \\
\hline Gestational age (wk) & $38.9 \pm 1.3$ & $39.0 \pm 1.6$ & $39.0 \pm 1.5$ & 0.55 \\
\hline $\begin{array}{l}\text { Head } \\
\text { circumference }(\mathrm{cm})\end{array}$ & $34.7 \pm 1.5$ & $34.7 \pm 1.8$ & $34.7 \pm 1.7$ & 0.98 \\
\hline Gender: female & $30(48 \%)$ & $37(51 \%)$ & $67(50 \%)$ & 0.87 \\
\hline Apgar score at $5 \mathrm{~min}$ & $2.7 \pm 2.2$ & $2.8 \pm 1.9$ & $2.8 \pm 2.0$ & 0.81 \\
\hline $\begin{array}{l}\text { aEEG background: } \\
\text { severe }\end{array}$ & $19(26 \%)$ & $20(32 \%)$ & $39(29 \%)$ & 0.71 \\
\hline Clinical HIE: severe & $16 / 60(27 \%)$ & $11 / 73(15 \%)$ & $27(20 \%)$ & 0.13 \\
\hline $\begin{array}{l}\text { Age at } \\
\text { randomization (h) }\end{array}$ & $4.59 \pm 0.82$ & $4.60 \pm 0.88$ & $4.59 \pm 0.85$ & 0.99 \\
\hline
\end{tabular}

aEEG, amplitude integrated electroencephalogram; HIE, hypoxic-ischemic encephalopathy.

and Tobin regression confirmed that the relationship was not affected by censoring (Figure 3). Favorable outcome at 18 mo predicted overall WeeFIM ratings within the normal range at $7-8$ y $(P=0.001$, Fisher's exact test $)$ with a sensitivity of 0.87 (95\% confidence interval (CI): $0.77-0.93$ ), specificity of 0.56 (95\% CI: $0.42-0.66)$, positive predictive value of $0.74(95 \%$ CI: $0.66-0.80$ ), and negative predictive value of 0.74 (95\% CI: $0.55-0.87)$. Favorable primary outcome at 18 mo predicted normal WeeFIM mobility $(P<0.0001)$ with a sensitivity of 0.87 (95\% CI: $0.80-0.91$ ), specificity of 0.81 (95\% CI: $0.62-$ 0.93 ), positive predictive value of 0.93 (95\% CI: $0.86-0.97$ ), and negative predictive value of 0.68 (95\% CI: $0.52-0.78$ ) (Figure 4).

There was no significant effect of treatment on WeeFIM ratings $(P=0.83)$.
Table 2. Primary outcome for tested and untested survivors assessed at $18 \mathrm{mo}$

\begin{tabular}{lcc}
\hline & $\begin{array}{c}\text { Tested } \\
(N=62)\end{array}$ & $\begin{array}{c}\text { Not tested } \\
(N=73)\end{array}$ \\
\hline $\begin{array}{l}\text { Unfavorable primary outcome } \\
\text { at } 18 \text { mo }\end{array}$ & $19(31 \%)$ & $30(40.5 \%)$ \\
GMF score & $1.1 \pm 1.9$ & $1.6 \pm 2.1$ \\
Severe neuromotor disability & $10(16 \%)$ & $22(30 \%)$ \\
GMF $\geq 3)$ & & \\
Bayley MDI score & $81.2 \pm 20.9$ & $78.2 \pm 24.0$ \\
MDI <70 & $17(28 \%)$ & $24(35 \%)$ \\
Bayley PDI score & $79.9 \pm 21.5$ & $79.9 \pm 24.4$ \\
Bilateral cortical visual impairment & $5(8 \%)$ & $11(15 \%)$ \\
Bilateral hearing loss & $6(11 \%)$ & $2(3 \%)$ \\
Epilepsy & $5(8 \%)$ & $15(20 \%) / 59$ \\
Cerebral palsy & $21(34 \%)$ & $28(38 \%)$ \\
Multiple handicaps & $10(18 \%)$ & $22(30 \%)$ \\
\hline
\end{tabular}

GMF, gross motor function; MDI, mental development index; PDI, physical developmental index.

\section{DISCUSSION}

This is the first report of neurodevelopmental outcomes at school age in children treated as infants with therapeutic hypothermia. As discussed next, the current study was not able to determine whether treatment affected long-term outcome. However, measured outcome at 18 mo was strongly associated with overall WeeFIM ratings at 7-8 y of age, supporting the validity of previous conclusions of a sustained treatment effect of therapeutic hypothermia for neonatal encephalopathy $(3,4)$.

The major challenge facing this study was that the original CoolCap study was powered to detect a difference in death 
Table 3. Baseline characteristics of follow-up study population

\begin{tabular}{lccc}
\hline & $\begin{array}{c}\text { Cooled } \\
(N=32)\end{array}$ & $\begin{array}{c}\text { Control } \\
(N=30)\end{array}$ & $P$ value \\
\hline Birth weight $(\mathrm{g})$ & $3,286 \pm 617$ & $3,492 \pm 771$ & 0.25 \\
Gestational age $(\mathrm{wk})$ & $39.0 \pm 1.3$ & $38.7 \pm 1.4$ & 0.33 \\
Head circumference $(\mathrm{cm})$ & $34.5 \pm 1.7$ & $34.8 \pm 1.3$ & 0.59 \\
Gender: female & $12(38 \%)$ & $18(60 \%)$ & 0.13 \\
Apgar score at 5 min & $2.4 \pm 2.1$ & $3.0 \pm 2.2$ & 0.91 \\
aEEG background: severe & $11(34 \%)$ & $9(30 \%)$ & 0.79 \\
Clinical HIE: severe & $12(38 \%)$ & $4(13 \%)$ & 0.08 \\
Age at randomization (h) & $4.6 \pm 0.9$ & $4.6 \pm 0.7$ & 0.80 \\
Age at follow-up (mo) & $92.2 \pm 7.4$ & $91.9 \pm 7.6$ & 0.69 \\
\hline aEEG amplitude integrated electroencephalogram; HIE, hypoxic-ischemic \\
encephalopathy.
\end{tabular}

Table 4. Concordance between WeeFIM ratings (individual domains and total rating) at 7-8y and neurodevelopmental outcome at $18 \mathrm{mo}$

\begin{tabular}{lcc}
\hline & $\begin{array}{c}\text { Number (\%) with } \\
\text { normal WeeFIM rating } \\
\text { and favorable outcome } \\
\text { at 18 mo }(n=43)\end{array}$ & $\begin{array}{c}\text { Number (\%) with } \\
\text { abnormal WeeFIM } \\
\text { rating and unfavorable } \\
\text { outcome at 18 mo } \\
(n=19)\end{array}$ \\
\hline Self-care & $33(77 \%)$ & $14(74 \%)$ \\
Mobility & $40(93 \%)$ & $13(68 \%)$ \\
Cognition & $32(74 \%)$ & $13(68 \%)$ \\
Total WeeFIM rating & $32(74 \%)$ & $14(74 \%)$ \\
\hline
\end{tabular}

Data are $N(\%)$.

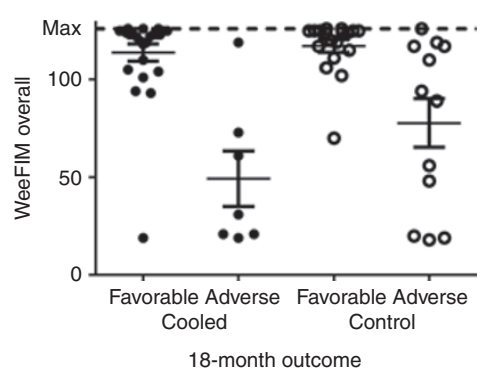

Figure 2. WeefIM ratings as a function of outcome at $18 \mathrm{mo}$ of age. There was no significant effect of treatment on WeeFIM ratings, but outcome at 18 mo was strongly predictive of normal vs. abnormal WeeFIM scores at $7-8$ y $(P<0.001$, logistic regression). Horizontal bars show the median and 25th and 75th percentiles.

or disability at $18 \mathrm{mo}$, and the study had limited power to detect a difference in subsequent disability in survivors, as described under Methods. With approximately a further 50\% loss to follow-up, the present study had insufficient power to examine whether hypothermia improved functional outcomes. Thus, the major focus of this study was to assess the predictive value of outcome at 18 mo using a binary outcome to determine whether the original binary outcome was still valid at 7-8 y. Reassuringly, we found that a favorable outcome in infancy was highly associated with normal functional outcomes at school age. This finding suggests that these

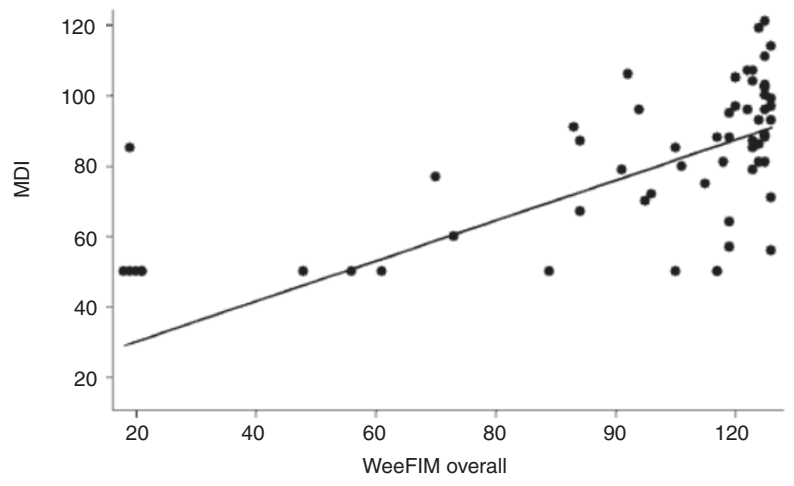

Figure 3. MDI at $18 \mathrm{mo}$ vs. overall WeeFIM rating. The solid line shows the linear relationship between $\mathrm{MDI}$ assessed at $18 \mathrm{mo}$ and the overall WeeFIM rating assessed at 7-8 y. MDI, Bayley Mental Development Index.

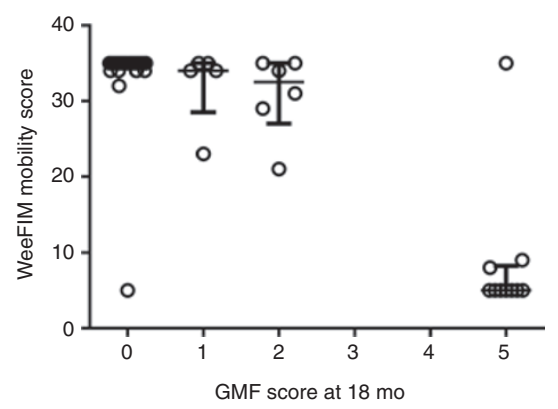

Figure 4. WeeFIM mobility subdomain at 7-8 y of age vs. gross motor function (GMF) score at 18-22 mo of age. There is a strong association between longer term motor outcomes and motor outcomes determined at 18-22 mo of age. Similarly, cerebral palsy diagnosed at $18-22$ mo of age is highly associated with the WeeFIM mobility score at 7-8y (data not shown).

early assessments reliably predict good functional outcomes at school age. It was of interest that the mobility scores correlated better than other subdomains with the 18-mo outcome; this likely reflects the predominance of neuromotor disabilities in infancy (9). Alternatively, therapeutic hypothermia may serve to preserve motor function better than cognitive function or the 18-mo Bayley assessment is a less valid predictor of long-term cognitive outcomes, because cognitive testing at this young age is difficult. The comparability of the baseline characteristics of the population available for follow-up and those not interviewed suggests that the results would likely not change if the entire population were able to be studied.

Previous long-term follow-up studies suggest that survivors of neonatal encephalopathy without major disability typically have an increased risk of subtle neurological disabilities when they are assessed at school age. In contrast, consistent with the present study, severe disability diagnosed early in life is persistent (7-10), and the severity of disability appears to be proportional to the severity of neural injury in the neonatal period and in childhood $(11,12)$. For example, in one cohort of 53 children born at term with encephalopathy, $36 \%$ had cerebral palsy; of the 34 remaining infants, at school age $8(15 \%)$ had minor neurological dysfunction and/or perceptual-motor 
difficulties, 1 (2\%) had only cognitive impairment, and 25 (47\%) were normal (8).

In the present study, there was a wide spread of ratings among the children with abnormal assessments at 18 mo when the families were questioned when children were at school age. It is difficult, in the absence of in-person testing or school reports, to be certain whether these children are truly within normal range for age or whether, perhaps, both the family and the child may have learned to employ compensatory skills to allow them to function adequately in activities of daily living (13). Thus, the results of the evaluation at 18 mo may be most useful for reassurance for parents of children who are doing well at that age and to target children for whom intervention may improve functional status later on. In turn, the WeeFIM instrument may be helpful as a screening tool in older children to allow the most appropriate use of limited resources, both to target children at greatest need for services and to identify children for whom more specific testing may be indicated.

The present study has several limitations that should be considered, including incomplete follow-up and the absence of inperson evaluations by neutral observers. Because participating families lived across the United States, in the United Kingdom, and in New Zealand and because each participating site had only a small number of children, we evaluated survivors using a validated tool that could be administered by a single individual over the phone to willing families, allowing greater consistency and effective masking of the examiner to initial presentation and to study arm assignment. The WeeFIM instrument is a wellvalidated tool for assessing the functional status of children at early school age in several domains of daily life in children with a wide range of abilities, from independently functioning to needing total assistance (14). Because several children in this study were expected to be extremely disabled, one of the major advantages of this instrument was that its descriptive range extends to the most functionally impaired children.

In summary, we provide initial data that the follow-up evaluation at 7-8 y of age in children initially assessed at $18 \mathrm{mo}$ is relatively stable over time both in the cooled and in the noncooled group. In particular, toddlers who are appropriate for age are likely to continue to show normal overall functional outcome to school age. Thus, although this study had insufficient power to directly assess the impact of therapeutic hypothermia, this finding supports an association between a favorable outcome at 18 mo assessed by published trials of therapeutic hypothermia and a favorable outcome at 7-8 y.

\section{METHODS}

\section{Subjects}

Of the original 234 infants enrolled in the CoolCap Trial, 140 of the 156 (90\%) children who survived had clinical neurological examination and were assessed with the Bayley Scales of Infant Development II at $18 \mathrm{mo}$ of age (Figure 1). These children were located in the United Kingdom (15), New Zealand (5), and Canada (11) and at 25 sites in 12 different states in the United States. Of these, 135 children survived to 7-8 y of age. Because of privacy issues, the principal investigator at each of the sites who agreed to participate in this follow-up protocol (21 of 25) contacted the families and obtained informed consent and agreement to the phone interview that was then conducted by a central certified examiner. This study was approved by the institutional review board of the University of Rochester Medical Center and by the institutional review boards of all participating institutions.

\section{Measures}

Given the wide geographic dispersal of these children and the wide range of their cognitive and motor abilities, parents were interviewed by phone using the WeeFIM instrument (the Functional Independence Measure for Children, WeeFIM, Uniform Data System for Medical Rehabilitation, Buffalo, NY) (15). The interviews were conducted by a single trained and certified interviewer to minimize interexaminer variability of administration and scoring. The WeeFIM II may be used for ages 6 mo to $7 \mathrm{y}$; it may also be used for children above the age of $7 \mathrm{y}$ as long as their functional abilities (as measured by this instrument) are below those expected of 7-year-olds who do not have disabilities. The measure consists of a set of ratings of 18 skills divided into three general domains: 8 of self-care (e.g., eating, dressing upper body), 5 of mobility (e.g., transfer to chair/wheelchair, walk), and 5 of cognition (comprehension, expression, social interaction, problem solving, and memory) (15). Caregivers are asked to rate a child about the extent of independence, full functioning, in carrying out each of those 18 skills, rated on a scale from 1 for total assistance, total dependence, maximal prompting, or not testable to 7 for complete independence. The ratings are combined to yield three domain scores and a WeeFIM total. The three domain scores can range from 8 to 56 for self-care, 5 to 35 for mobility, and also 5 to 35 for cognition. The total score for all 18 skills can range from 18 to 126.

In the CoolCap Trial, adverse outcomes in survivors were defined as Bayley Mental Development Index $<70$ or Gross Motor Function Classification $3-5$ or bilateral cortical visual impairment (4). For the present study, abnormal function at $7-8$ y of age was defined as WeeFIM scores less than one standard deviation below the mean for age for normal children, for both the total score and the three domain scores (15); thus, for example, a total WeeFIM score of $>106$ was defined as normal.

\section{Statistical Analysis}

For the primary analysis, logistic regression was used to assess, first, whether favorable 18-mo status as defined in the CoolCap study (4) was predictive of normal 7- to 8-y WeeFIM status and then, second, whether hypothermia treatment was associated with WeeFIM status. For assessment of the effect of treatment, the modified Sarnat hypoxic-ischemic encephalopathy stage, severity of amplitude integrated electroencephalogram background abnormality, and 5-min Apgar scores were included as covariates consistent with the analysis of the CoolCap study $(4,5)$. As a supplementary analysis, because the 18 -mo outcomes were censored because of the nature of the test, we carried out a Tobin censored regression to confirm that our conclusions were not affected by the censoring.

\section{Power to Detect an Effect on the Primary Outcomes}

The rate of favorable outcome among survivors of the CoolCap study at 18 mo of age was $68 \%$ in the cooled group and $54 \%$ in the noncooled group (4). With 135 subjects, the study would have approximately a $40 \%$ power to detect a comparable $14 \%$ difference in the incidence of WeeFIM scores $>-1$ SD between treatment groups; with 62 subjects, the study had $20 \%$ power to detect such a difference. In contrast, the study had much greater power to study the predictive value of the 18 -mo examination. If $50 \%$ of the subjects had favorable outcomes at $7 \mathrm{y}$, all from the 18-mo favorable group, with no crossover from the 18-mo unfavorable group, with a $30 \%$ loss rate, the power to detect a statistically significant association between status at $18-\mathrm{mo}$ and $7-\mathrm{y}$ is $89 \%$ at a two-sided 0.05 significance level.

\section{ACKNOWLEDGMENTS}

The authors acknowledge the efforts of Joan Merzbach, who contacted all the families, site investigators, and coordinators and performed the interviews. 
The following investigators participated in the CoolCap Study Group.

Executive committee: P.D. Gluckman (chair, co-principal investigator), J.S. Wyatt (co-principal investigator), A.J. Gunn (Scientific Officer).

Scientific advisory committee: J.S. Wyatt (chair), R. Ballard, A.D. Edwards, D.M. Ferriero, P.D. Gluckman, A.J. Gunn, R. Polin, C. Robertson, A. Whitelaw.

Data safety committee: R. Soll (chair), M. Bracken, C. Palmer, M. Heymann, A. Wilkinson.

Hospital investigators: J. Kaiser (Arkansas Children's Hospital, 9 patients with follow-up data), M. Battin, D. Armstrong (University of Auckland, National Women's Hospital, NZ, 4 patients), J. Khan (Children's Memorial Hospital and Prentice Women's Hospital of Northwestern Memorial Hospital, 0 patients), T. Raju (University of Illinois at Chicago Medical Center, 0 patients), R. Polin, R. Sahni, U. Sanocka (Children's Hospital of New York-Presbyterian, Columbia University, 3 patients), A. Rosenberg, J. Paisley (Children's Hospital of Denver, 8 patients), R. Goldberg, M. Cotton (Duke University Medical Center, 2 patients), D. Azzopardi, A.D. Edwards (Hammersmith Hospital, London, UK, 1 patient), J. Barks, S. Donn (University of Michigan Medical Center, Mott Children's Hospital, 3 patients), B. Couser (Children's Hospital and Clinics of Minneapolis, 7 patients), D. Durand (Children's Hospital and Research Center at Oakland, 0 patients), S. Adeniyi-Jones (AI Dupont Children's Hospital at Thomas Jefferson University Medical Center, 1 patient), T. Yanowitz (Magee Women's Hospital/ Children's Hospital of Pittsburgh, 5 patients), R. Guillet, N. Laroia (Golisano Children's Hospital at Strong, 4 patients), N. Finer, F. Mannino (University of California San Diego Medical Center (Hillcrest), 1 patient), J. Partridge (University of California San Francisco Children's Hospital, 1 patient), D. Davidson (Schneider Children's Hospital, 1 patient), A. Whitelaw (Southmead Hospital, Bristol, UK, 6 patients), M. Thoresen (St. Michael's Hospital, Bristol, UK, 4 patients), J.S. Wyatt, F. O'Brien (University College Hospital, London, UK, 0 patients), B. Walsh (Vanderbilt Children's Hospital, 2 patients), J. Perciaccante, M. O'Shea (Wake Forest University Baptist Medical Center, 1 patient).

Manufacturer's representatives: J. Jones, T. Weiler, J. Mullane, D. Hammond, J. Parnell (Olympic Medical (now Natus Medical), Seattle, WA).

The use of the WeeFIM instrument to collect data for this clinical trial was authorized and conducted in accordance with the terms of a special-purpose license granted to Licensee by Uniform Data System for Medical Rehabilitation, a division of UB Foundation Activities (UDSMR). The patient data collected during the course of this clinical trial have not been processed by the UDSMR. No implication is intended that such data have been or will be subjected to the UDSMR's standard data-processing procedures.

WeeFIM is a trademark of Uniform Data System for Medical Rehabilitation, a division of UB Foundation Activities, Inc.

STATEMENT OF FINANCIAL SUPPORT

The study was sponsored by Olympic Medical (Seattle, WA).

\section{REFERENCES}

1. Perlman JM. Intrapartum hypoxic-ischemic cerebral injury and subsequent cerebral palsy: medicolegal issues. Pediatrics 1997;99:851-9.

2. Lawn JE, Cousens S, Zupan J. 4 million neonatal deaths: when? Where? Why? Lancet 2005;365:891-900.

3. Edwards AD, Brocklehurst P, Gunn AJ, et al. Neurological outcomes at 18 months of age after moderate hypothermia for perinatal hypoxic ischaemic encephalopathy: synthesis and meta-analysis of trial data. BMJ 2010;340:c363.

4. Gluckman PD, Wyatt JS, Azzopardi D, et al. Selective head cooling with mild systemic hypothermia after neonatal encephalopathy: multicentre randomised trial. Lancet 2005;365:663-70.

5. Wyatt JS, Gluckman PD, Liu PY, et al. Determinants of outcomes after head cooling for neonatal encephalopathy. Pediatrics 2007;119:912-21.

6. Higgins RD, Raju TN, Perlman J, et al. Hypothermia and perinatal asphyxia: executive summary of the National Institute of Child Health and Human Development workshop. J Pediatr 2006;148:170-5.

7. Marlow N, Rose AS, Rands CE, Draper ES. Neuropsychological and educational problems at school age associated with neonatal encephalopathy. Arch Dis Child Fetal Neonatal Ed 2005;90:F380-7.

8. Barnett A, Mercuri E, Rutherford M, et al. Neurological and perceptualmotor outcome at 5 - 6 years of age in children with neonatal encephalopathy: relationship with neonatal brain MRI. Neuropediatrics 2002;33:242-8.

9. Robertson CM. Long-term follow-up of term infants with perinatal asphyxia. In: Stevenson DK, Sunshine P, eds. Fetal and Neonatal Brain Injury. Cambridge, UK: Cambridge University Press, 2002:829-858.

10. Lindström K, Lagerroos P, Gillberg C, Fernell E. Teenage outcome after being born at term with moderate neonatal encephalopathy. Pediatr Neurol 2006;35:268-74.

11. Belet N, Belet U, Incesu L, et al. Hypoxic-ischemic encephalopathy: correlation of serial MRI and outcome. Pediatr Neurol 2004;31:267-74.

12. van Kooij BJ, van Handel M, Nievelstein RA, Groenendaal F, Jongmans MJ, de Vries LS. Serial MRI and neurodevelopmental outcome in 9- to 10-year-old children with neonatal encephalopathy. J Pediatr 2010; 157:221-227.e2.

13. Lindsay NM, Healy GN, Colditz PB, Lingwood BE. Use of the Ages and Stages Questionnaire to predict outcome after hypoxic-ischaemic encephalopathy in the neonate. J Paediatr Child Health 2008;44:590-5.

14. Chen CC, Bode RK, Granger CV, Heinemann AW. Psychometric properties and developmental differences in children's ADL item hierarchy: a study of the WeeFIM instrument. Am J Phys Med Rehabil 2005;84:671-9.

15. Msall ME, DiGaudio K, Rogers BT, et al. The Functional Independence Measure for Children (WeeFIM). Conceptual basis and pilot use in children with developmental disabilities. Clin Pediatr (Phila) 1994;33:421-30. 